\title{
Intravenous cangrelor as a peri-procedural bridge with applied uses in ischemic events
}

\author{
Venugopal B. Bhattad ${ }^{1}$, Sathvika Gaddam², Margaret A. Lassiter ${ }^{3}$, Pooja S. Jagadish ${ }^{4}$, Devarshi Ardeshna ${ }^{5}$, \\ Brandon Cave $^{6}$, Rami N. Khouzam ${ }^{7}$
}

${ }^{1}$ Department of Internal Medicine, Division of Cardiovascular Diseases, ${ }^{2}$ Department of Internal Medicine, East Tennessee State University, Johnson City, TN, USA; ${ }^{3}$ Cardiovascular Clinical Pharmacy Department, Johnson City Medical Center, Johnson City, TN, USA; ${ }^{4}$ Department of Internal Medicine, ${ }^{5}$ College of Medicine, University of Tennessee Health Science Center, Memphis, TN, USA; ${ }^{6}$ Department of Pharmacy, Methodist University Hospital, Memphis, TN, USA; ${ }^{7}$ Department of Internal Medicine, Division of Cardiovascular Diseases, University of Tennessee Health Science Center, Memphis, TN, USA

Contributions: (I) Conception and design: VB Bhattad, B Cave, D Ardeshna, PS Jagadish; (II) Administrative support: RN Khouzam; (III) Provision of study materials or patients: All authors; (IV) Collection and assembly of data: VB Bhattad, S Gaddam, MA Lassiter, D Ardeshna; (V) Data analysis and interpretation: VB Bhattad, S Gaddam, MA Lassiter, D Ardeshna, PS Jagadish; (VI) Manuscript writing: All authors; (VII) Final approval of manuscript: All authors.

Correspondence to: Venugopal B. Bhattad. Department of Internal Medicine, Division of Cardiovascular Disease, Quillen College of Medicine, East Tennessee State University, PO Box 76022, Johnson City, TN 37614, USA. Email: venubrij@gmail.com.

\begin{abstract}
Cangrelor is a relatively new antiplatelet drug that has been approved for use as an adjunct therapy to percutaneous coronary intervention (PCI) to decrease peri-procedural myocardial infarction (MI), coronary revascularization, and stent thrombosis. Cangrelor is an adenosine triphosphate analogue with a pharmacokinetic mechanism based on a reversible, dose-dependent inhibition adenosine diphosphate (ADP)induced platelet aggregation. This drug has lately been in the spotlight as a possible bridge therapy for antiplatelet medication prior to cardiac and non-cardiac surgeries. Platelet function is usually restored within sixty minutes of cessation of therapy, thereby decreasing the risk of bleeding while providing adequate preprocedural coverage to reduce ischemic events. This manuscript reviews the literature on cangrelor and summarizes its role as a peri-procedural bridge.
\end{abstract}

Keywords: Cangrelor; percutaneous coronary intervention (PCI); P2Y12; peri-procedural bridge

Submitted Jul 01, 2019. Accepted for publication Jul 15, 2019.

doi: $10.21037 /$ atm.2019.07.64

View this article at: http://dx.doi.org/10.21037/atm.2019.07.64

\section{Introduction}

Dual antiplatelet therapy (DAPT) is a central part of the medical management of patients with coronary artery disease (CAD), specifically those with recent acute coronary syndrome (ACS) events. DAPT includes aspirin plus a P2Y12 receptor inhibitor (e.g., clopidogrel, prasugrel, and ticagrelor) and must be continued for a time between one month and one year based on the patient's level of heart disease, stent type, and proximity to stent placement (1). Several studies have demonstrated that DAPT decreases the risk of stent thrombosis, and interruption of therapy may increase this risk depending on the time from stent placement (2). The 2014 American College of Cardiology/ American Heart Association (ACC/AHA) guideline for the management of patients with non-ST-elevation ACS recommends starting P2Y12 inhibitors as early as possible in patients with ACS. However, a significant number of these patients require urgent or emergent coronary artery bypass grafting (CABG) (3). Administration of an oral $\mathrm{P} 2 \mathrm{Y} 12$ inhibitor may delay surgical procedures to allow time for restoration of platelet function (4).

Given the need for DAPT in the early stages of ACS or PCI, there are two major, paradoxical concerns regarding 
platelet reactivity in the perioperative setting should a patient require emergent CABG. First, interruption of DAPTparticularly among recently-stented patients-has been shown to increase thrombotic risk among patients undergoing surgery $(5,6)$. Second, continuation of DAPT peri-operatively during $\mathrm{CABG}$ and non-cardiac surgery demonstrably increases the risk of major bleeding when compared to placebo (7). Acknowledging the increased risk of bleeding in patients undergoing surgery and the risk of ischemia and thrombosis attributable to suspension of antiplatelet therapy, a "bridge" with a rapid onset and offset of action would be an ideal strategy in the management of patients on antiplatelet therapy during the perioperative period.

Cangrelor is an intravenous (IV) P2Y12 inhibitor that has recently emerged as a potential therapy for these highrisk, peri-procedural patients. Studies on cangrelor have failed to demonstrate a significantly increased bleeding risk when P2Y12 inhibitors were stopped and IV cangrelor bridge therapy was used between five and seven days pre-procedurally (8). Additionally, cangrelor has been demonstrated to reduce peri-procedural myocardial infarctions (MIs) in the post-ACS population $(9,10)$. This highlights the potential role of this novel P2Y12 inhibitor among ACS patients.

\section{The guidelines on delaying surgery}

The 2011 American College of Cardiology Foundation (ACCF)/AHA Guideline for Coronary Artery Bypass Graft Surgery recommends pre-CABG washout period of five days for clopidogrel [class I, level of evidence (LOE) $\mathrm{B}$ ] and seven days for prasugrel (class I, LOE: C) due to the increased risk of bleeding events while on P2Y12 inhibitors (11). The 2014 ACC/AHA guideline for the management of patients with non-ST-elevation ACS further specifies that clopidogrel and ticagrelor must be stopped at least 24 hours prior to urgent CABG (class I, LOE: B) (3). This delay allows for the normalization of platelet function. Even ticagrelor, which binds reversibly to platelets must be discontinued at least three days prior to procedures due to its high potency for platelet inhibition $(11,12)$. Until recently, the mainstay of therapy has been glycoprotein IIb/IIIa inhibitors (GPI), namely eptifibatide and tirofiban. However, the development of cangrelor as a short-acting antiplatelet has created opportunities for treatment periprocedurally.

\section{Cangrelor}

\section{Benefits of cangrelor}

Cangrelor initially gained Food and Drug Administration (FDA) approval in December 2014 for patients undergoing percutaneous coronary intervention (PCI) who have not been treated with a P2Y12 platelet inhibitor and who are not being given a GPI. Approval of cangrelor for this indication was based primarily on the Effect of Platelet Inhibition with Cangrelor during PCI on Ischemic Events (CHAMPION PHOENIX) trial-a large multicenter, randomized, double-blind, placebo controlled trial with 11,145 patients undergoing either urgent or elective PCI while on guidelinerecommended medical therapy (9). Cangrelor achieved a marginal benefit over clopidogrel in the composite primary endpoint that was driven by a reduction in periprocedural MI $(9,10)$. Cangrelor has since been added for this indication to the 2018 European Society for Cardiology/European Association for Cardio-Thoracic Surgery (ESC/EACTS) guidelines on myocardial revascularization [2018] and the 2015 guidelines for the management of ACS in patients presenting without persistent ST-segment elevation $(13,14)$. However, it is not part of American guidelines as of yet.

\section{Pharmacology of cangrelor}

Cangrelor is a selective, rapidly reversible P2Y12 platelet receptor inhibitor that directly blocks adenosine diphosphate (ADP)-induced activation and aggregation of platelets and achieves a $90 \%$ level of platelet inhibition within five minutes and reaches steady state within fifteen minutes, based on the PCI dosing from the CHAMPION PHOENIX trial $(9,10)$. Due to its rapid onset and offsethaving a clinical half-life of five minutes-it allows for a quick, high degree of platelet inhibition and normalization of platelet activity sixty minutes after discontinuation $(10,15)$. In contrast, eptifibatide requires four to six hours for platelet function normalization after discontinuation, and tirofiban needs four to eight hours (16). Cangrelor requires an initial bolus because it has a large volume of distribution-between 3.68 to 3.9 liters (10). Because cangrelor does not need dose adjustments for age, sex, hepatic function, or renal function, it is advantageous over other antiplatelet agents (10). 


\section{Perioperative monitoring of cangrelor}

\section{VerifyNow}

Multiple platelet function assays are available that can measure platelet aggregation and inhibition $(17,18)$. The VerifyNow Assay is a point-of-care tool developed to rapidly measure an individual's response to antiplatelet agents (19). It measures the rate of platelet activation by $\mathrm{ADP}$ binding to the $\mathrm{P} 2 \mathrm{Y} 12$ receptor via light transmittance in whole blood (20-22). This assay is specific to the platelet $\mathrm{P} 2 \mathrm{Y} 12$ receptor and is expressed in $\mathrm{P} 2 \mathrm{Y} 12$ reaction units (PRUs). VerifyNow was used specifically to guide cangrelor dosing in the trials brought to the Food and Drug Administration $(9,23,24)$. However, VerifyNow is not without its limitations. Results can be affected by a variety of factors, including thrombocytopenia or anemia as well as the use of GPIs (25).

It is important to note that the target range for effective platelet inhibition to reduce the risk of ischemia and bleeding in the peri-operative setting has not been well established. The VerifyNow manufacturer suggests that normal platelet function can range from 194-419 PRU (22). Among the cangrelor trials, the target was 240 PRUs (8). Notably, other studies have titrated cangrelor to a goal $\leq 208$ PRUs or $\geq 85$ PRUs (26-28).

\section{The Bridging Antiplatelet Therapy with Cangrelor in Patients Undergoing Cardiac Surgery (BRIDGE) trial}

The BRIDGE trial is a prospective, randomized-controlled, multicenter trial conducted between 2009 and 2011 (8). The trial included 210 patients treated with an oral P2Y12 antagonist (either clopidogrel, prasugrel, or ticagrelor) following an ACS-event or coronary stent who were to undergo CABG. During the trial, oral P2Y12 antagonists were discontinued, and patients were randomly allocated into either an IV cangrelor or placebo group for at least 48 hours and discontinued one to six hours prior to surgery (8).

The BRIDGE trial was conducted to determine the appropriate dose to maintain the platelet reactivity and to maintain target levels of PRU $<240$ for all on-infusion time points measured over the bridging period (8). The optimal dose was determined by two cohorts. The first cohort used a dose of $0.5 \mathrm{mcg} / \mathrm{kg} / \mathrm{min}$ with a goal to attain a predefined platelet inhibition above $60 \%$ in $80 \%$ of the study population. The initial cohort did not meet the study endpoint, having a platelet inhibition above $60 \%$ in only $76.5 \%$ of the study population (8). Thus, the study investigators increased the infusion dose to $0.75 \mathrm{mcg} / \mathrm{kg} / \mathrm{min}$ in the second cohort, yielding a platelet inhibition above $60 \%$ in $95.5 \%$ of the study subjects (8).

Using the dosing strategy from the second cohort, stage two of the trial found for the primary efficacy endpoint that $98.8 \%$ of cangrelor-treated patients maintained target levels of PRU $<240$ over the bridging period, compared to $19 \%$ for placebo-group patients [odds ratio $=353 ; 95 \%$ confidence interval (CI): 45.6-2,728; $\mathrm{P}<0.0001]$. The average duration of use was around 72 hours and is the longest duration of any study using cangrelor. Secondary endpoints evaluated included bleeding and thrombotic events, none of which demonstrated a statistically significant difference between study and placebo groups. Particularly the incidence of excessive CABG-related bleeding was balanced between cangrelor (11.8\%) and placebo (10.4\%), suggesting that cangrelor can safely be used in the CABG setting (8).

Although, cangrelor seemed promising based on the results of the study, the study was limited in two ways. First, it was underpowered to assess the differences in bleeding event rates. Second, degree of platelet blockade affected the risk of ischemic events in cangrelor group compared to placebo. As stated earlier, although PRU is a perioperative tool by which one may be able to tailor therapy, it has not yet been validated for the purposes of clinical outcomes. Of note, this trial only included patients undergoing CABG and cannot be generalized to those undergoing non-cardiac procedures due to difference in bleeding risk (8). While cangrelor shows promise as an IV bridge to cardiac surgery, it has yet to become part of American guidelines.

\section{Other studies on cangrelor as a bridge}

Since its release, cangrelor has been increasingly published for use as a peri-procedural bridge in small, retrospective studies or case series as summarized in Table 1.

Kairouz et al. published a single-center experience of using cangrelor as an antiplatelet bridge in $27 \mathrm{CAD}$-patients undergoing cardiac and non- cardiac surgery (29). The infusion dose was $0.75 \mathrm{mcg} / \mathrm{kg} / \mathrm{min}$ and the median time of discontinuation of infusion prior to surgery was 6.75 hours. Two out of twenty-one subjects who underwent cardiac surgeries had major bleeding, but no bleeding events were reported among noncardiac surgery patients (29).

In 2016, Bowman et al. performed a retrospective analysis of eleven patients who underwent coronary artery 
Table 1 Comparison of bridge trial and other smaller retrospective studies that used cangrelor as bridging therapy for DAPT

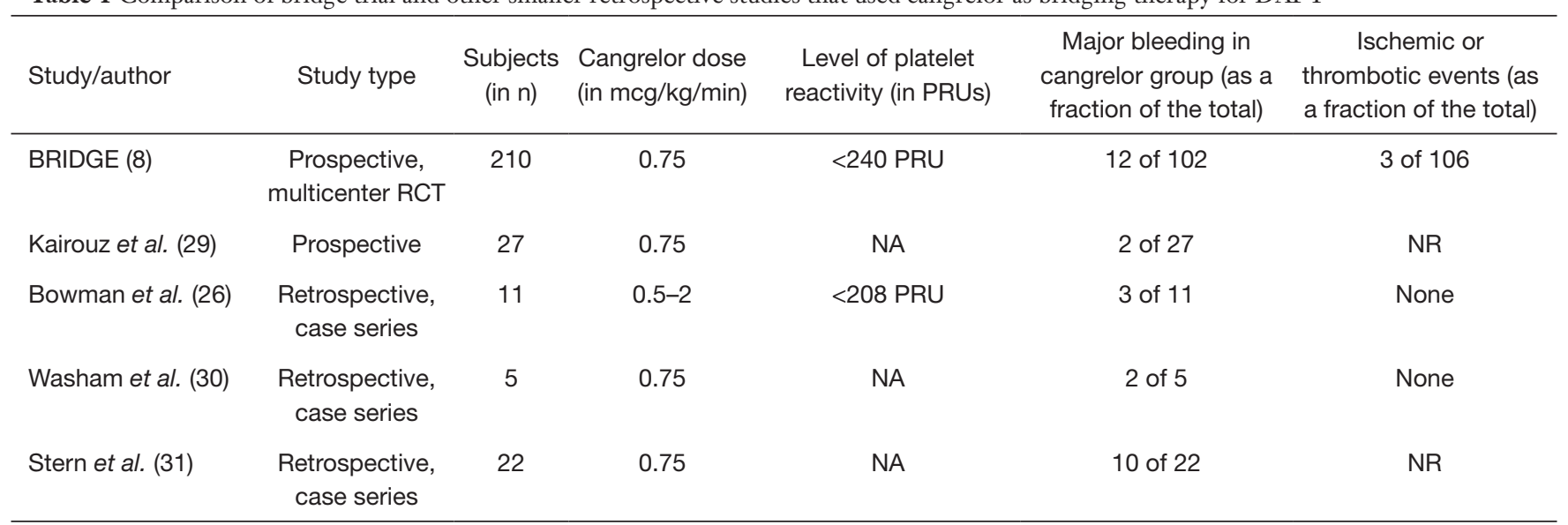

DAPT, dual antiplatelet therapy; RCT, randomized controlled trial; PRU, P2Y12 reaction unit; NR, not reported; NA, not applicable.

stenting and received cangrelor bridge therapy prior to cardiac surgery (26). The infusion dose was titrated between $0.5-2 \mathrm{mcg} / \mathrm{kg} / \mathrm{min}$ to maintain the platelet reactivity $<208$ PRU. The VerifyNow platelet reactivity assay was used to measure platelet function. The study reviewers suggested starting at low dose, $0.5 \mathrm{mcg} / \mathrm{kg} / \mathrm{min}$, to reduce the cost and risk of bleeding and titrate up if needed based on VerifyNow reactivity analysis (26).

Washam et al. presented a retrospective case series of five patients who received cangrelor as a bridge to left ventricular assist device (LVAD) implantation in those requiring DAPT for CAD (30). Two of the five patients had intrathoracic bleeding, but there were no ischemic events during the bridging period (30). Stern et al. published a retrospective case series of 22 patients who received cangrelor at a dose of $0.75 \mathrm{mcg} / \mathrm{kg} / \mathrm{min}$ (31). There were no reports of thrombosis, but ten of the 22 had major bleeding events (31).

\section{Perioperative bridging versus reversal}

Although bridging has been the recommended mainstay of antiplatelet management peri-operatively, on the other end of the spectrum, there is a new potential option on the horizon in the form of antiplatelet reversal. Currently named PB2452, this agent is a monoclonal antibody that binds ticagrelor with high affinity. The drug investigators recently published their phase I trial in healthy volunteers in March of 2019, showing that the agent provided immediate and sustained reversal of the antiplatelet effects of ticagrelor, as measured by VerifyNow P2Y12
Assay (32). Patients received 48 hours of ticagrelor therapy prior to administration of the reversal agent with an $80 \%$ suppression of platelet aggregation. Reversal of ticagrelor was attained within 5 minutes after the initiation of PB2452, and was sustained for more than 20 hours, with no evidence of rebound platelet activity after drug cessation.

This new drug may seem like an attractive option for perioperative management in the setting of ticagrelor pretreatment; however, there are some major concerns that would need to be addressed. Just as with anticoagulant therapy and reversal, immediate discontinuation of shortacting agents, like direct oral anticoagulants, or reversal of warfarin by means of vitamin $\mathrm{K}$ and activated fourfactor prothrombin complex concentrate, increases the risk of thromboembolic events $(33,34)$. Because of that risk, mitigation and reversal of these agents should be used judiciously in the setting of major, life-threatening bleeding or the need for an emergent procedure. Antiplatelet reversal, when available, should likely be used with the same caution to minimize the risk of in-stent thrombosis, while mitigating bleeding in the peri-procedural setting. Additionally, the reversal was sustained for upwards of 20 hours.

In direct comparison, cangrelor has shown transient recovery in platelet reactivity within the first hour after stopping the infusion (35). In the postoperative setting of cardiac or non-cardiac procedures, P2Y12 inhibitor therapy must be restarted as soon as possible with an appropriate loading dose (7). These competing agents would likely not allow for appropriate post-operative management of antiplatelet therapy to ensure thrombotic risk is minimized. 
Although a reversal agent might be beneficial in the setting of major, life-threatening bleeds, cangrelor might still hold a place for peri-procedural management of antiplatelet therapy where quick on and off-set antiplatelet activity may be more desirable.

\section{Conclusions}

Due to the increasing number of patients on antiplatelet therapy for cardiovascular and neurological conditions, it can be challenging to manage these patients perioperatively. It is critical to prevent ischemia and thrombosis, and at the same time decrease the risk of bleeding, necessitating the need for a successful bridging therapy. Cangrelor looks promising as a bridging therapy with its distinctive pharmacokinetic profile with fast activity and easy reversibility. However, large prospective studies are required to delineate clear guidelines to identify the patient population that would receive maximum benefit from bridging antiplatelet therapy, determine optimal dosing and titration, monitoring therapy, and manage adverse events. Although guidelines recommend IV bridge therapy in these settings, no agent currently has FDA-approval for this indication and positive, randomized controlled data for GPIs in this setting is also lacking. Cangrelor bridging therapy appears to have advantages over the previous standard using GPIs due to its faster offset and non-renal clearance (36). Future research is warranted for use of cangrelor in special populations, such as those with CAD on DAPT as a bridge to LVAD implantation. Overall, in this complex era of advancing medical technologies, therapies such as cangrelor may mitigate thrombotic and bleeding risks in the peri-operative period.

\section{Acknowledgments}

None.

\section{Footnote}

Conflicts of Interest: Dr. Cave discloses that he serves on the speaker's bureau for Portola pharmaceuticals. The other authors have no conflicts of interest to declare.

Ethical Statement: The authors are accountable for all aspects of the work in ensuring that questions related to the accuracy or integrity of any part of the work are appropriately investigated and resolved.

\section{References}

1. Levine GN, Bates ER, Bittl JA, et al. 2016 ACC/ AHA Guideline Focused Update on Duration of Dual Antiplatelet Therapy in Patients With Coronary Artery Disease. J Am Coll Cardiol 2016;68:1082.

2. Fox KA, Mehta SR, Peters R, et al. Benefits and risks of the combination of clopidogrel and aspirin in patients undergoing surgical revascularization for non-ST-elevation acute coronary syndrome: the Clopidogrel in Unstable angina to prevent Recurrent ischemic Events (CURE) Trial. Circulation 2004;110:1202-8.

3. Amsterdam EA WN, Brindis RG, Casey DE Jr, et al. 2014 AHA/ACC guideline for the management of patients with non-ST-elevation acute coronary syndromes: executive summary: a report of the American College of Cardiology/ American Heart Association Task Force on Practice Guidelines. Circulation 2014;130:2354-94.

4. Qamar A, Bhatt DL. Optimizing the Use of Cangrelor in the Real World. Am J Cardiovasc Drugs 2017;17:5-16.

5. Ferreira-González I, Marsal JR, Ribera A, et al. Double antiplatelet therapy after drug-eluting stent implantation: risk associated with discontinuation within the first year. J Am Coll Cardiol 2012;60:1333-9.

6. Collet JP, Montalescot G, Blanchet B, et al. Impact of prior use or recent withdrawal of oral antiplatelet agents on acute coronary syndromes. Circulation 2004;110:2361-7.

7. Levine GN, Bates ER, Bittl JA, et al. 2016 ACC/ AHA Guideline Focused Update on Duration of Dual Antiplatelet Therapy in Patients With Coronary Artery Disease: A Report of the American College of Cardiology/ American Heart Association Task Force on Clinical Practice Guidelines: An Update of the 2011 ACCF/AHA/ SCAI Guideline for Percutaneous Coronary Intervention, 2011 ACCF/AHA Guideline for Coronary Artery Bypass Graft Surgery, 2012 ACC/AHA/ACP/AATS/PCNA/ SCAI/STS Guideline for the Diagnosis and Management of Patients With Stable Ischemic Heart Disease, 2013 ACCF/AHA Guideline for the Management of STElevation Myocardial Infarction, 2014 AHA/ACC Guideline for the Management of Patients With NonST-Elevation Acute Coronary Syndromes, and 2014 ACC/AHA Guideline on Perioperative Cardiovascular Evaluation and Management of Patients Undergoing Noncardiac Surgery. Circulation 2016;134:e123-55.

8. Angiolillo DJ, Firstenberg MS, Price MJ, et al. Bridging antiplatelet therapy with cangrelor in patients undergoing 
cardiac surgery: a randomized controlled trial. Jama 2012;307:265-74.

9. Bhatt DL, Stone GW, Mahaffey KW, et al. Effect of platelet inhibition with cangrelor during PCI on ischemic events. N Engl J Med 2013;368:1303-13.

10. Baker DE, Ingram KT. Cangrelor. Hosp Pharm 2015;50:922-9.

11. Hillis LD, Smith PK, Anderson JL, et al. 2011 ACCF/ AHA Guideline for Coronary Artery Bypass Graft Surgery. A report of the American College of Cardiology Foundation/American Heart Association Task Force on Practice Guidelines. Developed in collaboration with the American Association for Thoracic Surgery, Society of Cardiovascular Anesthesiologists, and Society of Thoracic Surgeons. J Am Coll Cardiol 2011;58:e123-210.

12. Valgimigli M, Bueno H, Byrne RA, et al. 2017 ESC focused update on dual antiplatelet therapy in coronary artery disease developed in collaboration with EACTS: The Task Force for dual antiplatelet therapy in coronary artery disease of the European Society of Cardiology (ESC) and of the European Association for Cardio-Thoracic Surgery (EACTS). Eur Heart J 2018;39:213-60.

13. Neumann FJ, Sousa-Uva M, Ahlsson A, et al. 2018 ESC/ EACTS Guidelines on myocardial revascularization. Eur Heart J 2018;40:87-165.

14. Roffi M, Patrono C, Collet JP, et al. 2015 ESC Guidelines for the management of acute coronary syndromes in patients presenting without persistent ST-segment elevation: Task Force for the Management of Acute Coronary Syndromes in Patients Presenting without Persistent ST-Segment Elevation of the European Society of Cardiology (ESC). Eur Heart J 2016;37:267-315.

15. Akers WS, Oh JJ, Oestreich JH, et al. Pharmacokinetics and pharmacodynamics of a bolus and infusion of cangrelor: a direct, parenteral $\mathrm{P} 2 \mathrm{Y} 12$ receptor antagonist. J Clin Pharmacol 2010;50:27-35.

16. Luddington RJ. Thrombelastography/ thromboelastometry. Clin Lab Haematol 2005;27:81-90.

17. Price MJ. Bedside evaluation of thienopyridine antiplatelet therapy. Circulation 2009;119:2625-32.

18. Tantry US, Bonello L, Aradi D, et al. Consensus and update on the definition of on-treatment platelet reactivity to adenosine diphosphate associated with ischemia and bleeding. J Am Coll Cardiol 2013;62:2261-73.

19. van Werkum JW, Harmsze AM, Elsenberg EH, et al. The use of the VerifyNow system to monitor antiplatelet therapy: a review of the current evidence. Platelets 2008;19:479-88.
20. Elsenberg EH, van Werkum JW, van de Wal RM, et al. The influence of clinical characteristics, laboratory and inflammatory markers on 'high on-treatment platelet reactivity' as measured with different platelet function tests. Thromb Haemost 2009;102:719-27.

21. Breet NJ, van Werkum JW, Bouman HJ, et al. High ontreatment platelet reactivity to both aspirin and clopidogrel is associated with the highest risk of adverse events following percutaneous coronary intervention. Heart 2011;97:983.

22. Accumetrics. VerifyNow PRU'Test Platelet Reactivity Test Instructions For Use. Package Insert 2011.

23. Angiolillo DJ, Firstenberg MS, Price MJ, et al. Bridging Antiplatelet Therapy With Cangrelor in Patients Undergoing Cardiac Surgery: A Randomized Controlled TrialBridging to Surgery With Cangrelor. JAMA 2012;307:265-74.

24. Bhatt DL, Lincoff AM, Gibson CM, et al. Intravenous platelet blockade with cangrelor during PCI. N Engl J Med 2009;361:2330-41.

25. Pakala R, Waksman R. Currently available methods for platelet function analysis: advantages and disadvantages. Cardiovasc Revasc Med 2011;12:312-22.

26. Bowman S, Gass J, Weeks P. Antiplatelet Therapy Bridging With Cangrelor in Patients With Coronary Stents: A Case Series. Ann Pharmacother 2019;53:171-7.

27. Stone GW, Witzenbichler B, Weisz G, et al. Platelet reactivity and clinical outcomes after coronary artery implantation of drug-eluting stents (ADAPT-DES): a prospective multicentre registry study. Lancet 2013;382:614-23.

28. Campo G, Parrinello G, Ferraresi P, et al. Prospective evaluation of on-clopidogrel platelet reactivity over time in patients treated with percutaneous coronary intervention relationship with gene polymorphisms and clinical outcome. J Am Coll Cardiol 2011;57:2474-83.

29. Kairouz V, Patel P, Franchi F, et al. Cangrelor as an antiplatelet bridging strategy in patients with coronary artery disease undergoing cardiac and non-cardiac surgery. J Am Coll Cardiol 2018;71:A75.

30. Washam JB, Yerokun B, Patel CB, et al. Cangrelor use prior to left ventricular assist device surgery: a case series. J Thromb Thrombolysis 2018;46:131-3.

31. Stern G, Rimsans J, Qamar A, et al. Contemporary parenteral antiplatelet bridging strategies: a singlecentre real-world experience at a tertiary care centre. EuroIntervention 2018;14:e333-5.

32. Bhatt DL, Pollack CV, Weitz JI, et al. Antibody-Based 
Ticagrelor Reversal Agent in Healthy Volunteers. N Engl J Med 2019;380:1825-33.

33. Flaker GC, Theriot P, Binder LG, et al. Management of Periprocedural Anticoagulation: A Survey of Contemporary Practice. J Am Coll Cardiol 2016;68:217-26.

34. Patel IJ, Davidson JC, Nikolic B, et al. Consensus

Guidelines for Periprocedural Management of Coagulation Status and Hemostasis Risk in Percutaneous Image-guided
Interventions. J Vasc Interv Radiol 2012;23:727-36.

35. Schneider DJ, Seecheran N, Raza SS, et al.

Pharmacodynamic effects during the transition between cangrelor and prasugrel. Coron Artery Dis 2015;26:42-8.

36. Van Tuyl JS, Newsome AS, Hollis IB. Perioperative Bridging With Glycoprotein IIb/IIIa Inhibitors Versus Cangrelor: Balancing Efficacy and Safety. Ann Pharmacother 2019;53:726-37.
Cite this article as: Bhattad VB, Gaddam S, Lassiter MA, Jagadish PS, Ardeshna D, Cave B, Khouzam RN. Intravenous cangrelor as a peri-procedural bridge with applied uses in ischemic events. Ann Transl Med 2019;7(17):408. doi: 10.21037/atm.2019.07.64 